\title{
Atypical Presentation Of Paediatric Absence Seizure: EEG As A Diagnostic Tool
}

\author{
KC R $\mathbf{R}^{1}$, Poudel $\mathbf{R}^{\mathbf{2}}$ \\ 1. Lecturer, Department of Paediatrics, Nepalgunj Medical College, Kohalpur, Nepal 2. Assistant \\ Professor, Department of Psychiatry, Nepalgunj Medical College, Kohalpur, Nepal
}

E-mail *Corresponding author : rajeshkc75@gmail.com

\section{Abstract}

Absence seizures involve brief, sudden lapses of consciousness and are more common in children than in adults. We report a case of absence seizure in a girl with atypical presentation which was diagnosed by electroencephalogram. She responded well to sodium valproate. Detailed history, clinical examination and use of electroencephalogram for diagnosis is necessary especially when such atypical presentations are encountered.

Keywords: absence seizures, atypical presentation, electroencephalogram, spike and wave

\section{INTRODUCTION}

Seizure is a short episode of abnormal electrical activity in the brain with intense electrical signals. ${ }^{1}$ Absence seizures (AS) account for less than $10 \%$ of all seizure types and is one of the most common seizures to go undetected. ${ }^{2}$ It is usually idiopathic but can be less commonly caused by vascular malformation, infectious disease, neoplasm and toxic brain disease. ${ }^{3}$ These seizures are usually referred to the clinician with history of "staring spells" at home or in school. ${ }^{4}$ Typically, AS lasts from few seconds to minutes with the loss of consciousness and no any marked convulsions. It often occurs in children aged $<10$ years. ${ }^{5}$ AS can demonstrate behavioral arrest, unresponsiveness, cyclic blinking of eyelids, suddenly stopped speech and movement, mouth chewing, lip smacking, and rubbing of fingers. ${ }^{6}$ However, AS may also show unusual manifestations like episodes of throttling sensation and tightness of chest, loss of consciousness lasting 2-4 mins, giddiness, and dimness of vision. 2,5 Electroencephalogram (EEG) is the main diagnostic tool for absence seizures. Ethosuximide and sodium valproate are equally effective as monotherapy in controlling this type of seizure in more than $80 \%$ of children. ${ }^{7}$ We report a case of AS with atypical presentation in the form of preserved consciousness and awareness. During the episode the subject was unresponsive to verbal commands but moved backwards whenever someone tries to poke into her eyes in order to avoid injury. Diagnosis was later confirmed by EEG.

\section{CASE-REPORT}

Miss Sangini, seven years female from Bardiya, presented to the Pediatric Out-Patient Department with complaints of upward deviation of eyes for 3 months and was evaluated by the first author (RK). She experienced 10-12 such episodes daily, each episode lasting 10-30 seconds. There was no associated loss of consciousness, memory loss or sudden fall during the episodes. During most of the episodes, the patient was unresponsive to verbal commands but she moved backwards, whenever someone tries to poke into her eyes, in order to avoid injury. She also remembers and recognizes the person who had poked or pinched her during such episodes. She had no vomiting, involuntary movements, headache, fever, urinary incontinence, and palpitations.

There was no history of birth asphyxia, no similar history in the past, no head injury or any chronic illness. All developmental milestones were attained in time as per peer groups. There were no similar episodes in any family members. On clinical examination, the patient was conscious, co-operative, oriented, afebrile 
and with normal respiratory rate. Her pulse rate and blood pressure were below $90^{\text {th }}$ percentile for her age. No pallor/icterus/cyanosis/pedal edema was seen. The cardiovascular sounds S1 and S2 were normal and P/A was soft, no organomegaly.

Patient's central nervous system examination revealed normal higher mental functions with no motor as well as sensory deficits and bilateral flexor plantars. Normal developmental milestones as per age with good academic and extracurricular performance.

Complete blood picture, serum electrolytes and blood sugar test were reported to be normal. Patient was referred to the Department of Psychiatry and was evaluated by the second author (RP). She had no family history of any mental disorders and her mental status examination (MSE) was normal. EEG of brain and Computed Tomography (CT) of Head were advised. Patient developed a similar episode of eyes deviation while performing EEG. EEG showed $3 \mathrm{~Hz}$ spike-and-wave complexes confirming the detection of AS (Fig 1). CT Head showed normal scan.

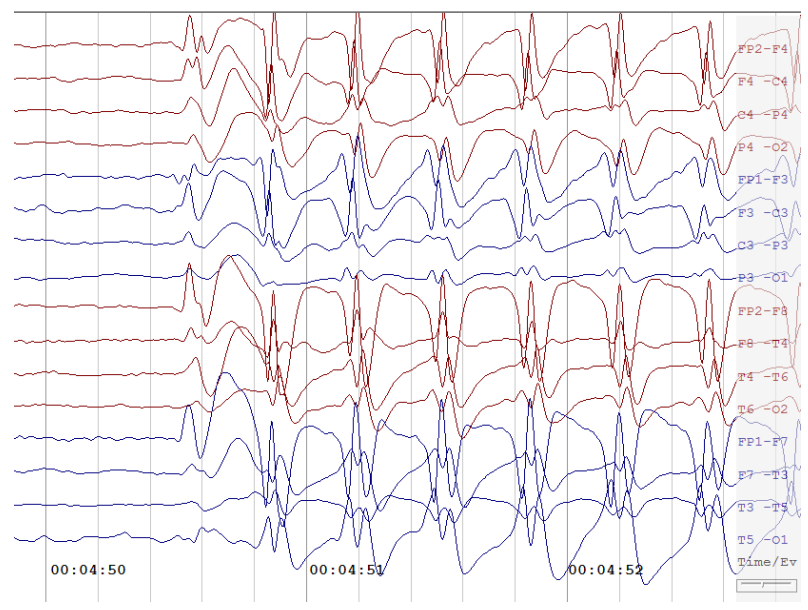

Fig 1. EEG of the Brain

She was diagnosed with AS and started on oral sodium valproate at $15 \mathrm{mg} / \mathrm{kg} /$ day. In clinical follow-up after one week, the patient was found responding to treatment, with decrease in frequency as well as duration of such eye deviation episodes. On one month follow-up, patient did not have any similar episodes. Follow-up EEG at one month was also normal.

\section{DISCUSSION:}

AS occurs in multiple genetic generalized epilepsies including childhood absence epilepsy (CAE), juvenile absence epilepsy (JAE), and juvenile myoclonic epilepsy (JME). ${ }^{8}$ CAE is a common pediatric epilepsy syndrome. The age of onset for CAE is typically between 4 and 10 years, with a peak between 5 and 7 years. Girls have CAE more frequently than boys. ${ }^{9}$ Absence status consists of generalized, non-convulsive seizures characterized by impairment of awareness, and intermittently has other manifestations such as automatisms or subtle myoclonic, tonic, atonic, or autonomic phenomena. ${ }^{10}$ Although AS occurs spontaneously, sometimes, environmental triggers influence it, especially emotional lability and such episodes are often mistaken as psychiatric symptoms. EEG is the main diagnostic tool for AS which was utilised in our case too. ${ }^{11}$ Although not life threatening, AS may lead to poor performances in school if left untreated. The primary drugs are sodium valproate, ethosuximide and lamotrigine. The duration of therapy is variable, although the general rule is to taper the therapy after seizure free period of two years. Typical AS generally have a favorable prognosis with remission rates as high as $80-90 \% .^{12}$ Therefore, any such abnormal behavioral aberration must be recognized and investigated accordingly.

As described in this case, AS can present with atypical features which many times can go unnoticed. A child may not be able to describe his/her absence symptoms to their parents or teachers and this may delay in visiting a clinician. In such situations it becomes difficult to diagnose absence seizure as a proper history may not be available. So cases like these especially with atypical features, need detailed history, clinical examination and use of EEG as a diagnostic tool for diagnosis and management.

\section{CONCLUSION:}

As observed in this report, AS can present with features that can pose a diagnostic dilemma. A multi-departmental approach including pediatrician, psychiatrist, and neurologist (whenever available) with utilization of EEG is necessary to diagnose and manage this disorder. 


\section{REFERENCES:}

1. Shah AM, Vashi A, Jagoda A. Convulsive and non-convulsive status epilepticus: An emergency medicine perspective. Emergency Medicine Australasia. 2009;21(5):352-66.

2. Ahmed R, Varghese T. Unusual presentation of absence seizures: A case report. Journal of Pediatric Neurology. 2015;04(01):45-7.

3. Femi OL, Sale S. Absence seizure in the elderly. Annals of African Medicine. 2011;10(2):188-91.

4. Holmes GL, McKeever M, Adamson M. Absence seizures in children: clinical and electroencephalographic features. Annals of Neurology. 1987;21(3):268-73.

5. Umashankar MS, Kumar AB. Clinical case presentation on absence seizures diagnosis and treatment care services and outcomes in an adult patient. Journal of neurosciences in rural practice. 2019;10(1):154-7.

6. Genton P, Ferlazzo E, Thomas P. Absence status epilepsy: Delineation of a distinct idiopathic generalized syndrome. Epilepsia. 2008;49:642-9.

7. Panayiotopoulos CP. Typical absence seizures and their treatment. Archives of disease in childhood. 1999;81(4):351-5.

8. Unterberger I, Trinka E, Kaplan PW, Walser G, Luef $G$, Bauer G. Generalized nonmotor (absence) seizures - What do absence, generalized, and nonmotor mean? Epilepsia. 2018;59(3):523-9.

9. Pearl PL. Epilepsy Syndromes in Childhood. Continuum (Minneap Minn). 2018;24(1, Child Neurology):186-209.

10. Genton P, Ferlazzo E, Thomas P. Absence status epilepsy: delineation of a distinct idiopathic generalized epilepsy syndrome. Epilepsia. 2008;49(4):642-9.

11. E. Faught, Clinical trials for treatment of primary generalized epilepsies, Epilepsia. 2003; 4(7):44-50.

12. Huguenard $J R$, Prince DA. Intrathalamic rhythmicity studied in vitro: nominal T-current modulation causes robust antioscillatory effects. Journal of Neuroscience. 1994;14(9):5485-502. 